\title{
Review
}

\section{The late stages of autophagy: how does the end begin?}

\author{
T Noda ${ }^{1}$, N Fujita ${ }^{1}$ and T Yoshimori ${ }^{*, 1}$
}

\begin{abstract}
Autophagy is a catabolic cellular process involving dynamic membrane rearrangement. Here, we review the understanding of autophagy, focusing on the late stages of the process, from the closing of the autophagosome to fusion with the lysosome. We propose the Reverse fusion model, for the closing autophagosome. In this model, autophagosome closure proceeds in a topologically similar but reverse order to membrane fusion during the escape of influenza virus from the endosome. This dynamic process is thought to be directly catalyzed by $\mathrm{LC} 3$, an ubiquitin-like molecule. Further, we discuss the dynamics of the Atg16L complex in relation to the LC3 localization in these processes. Finally, the molecular mechanisms involved in the delivery of autophagosomes to the lysosome and fusion are introduced. Several key events exist in each step and seem to be coordinated to faithfully conduct the autophagic process.
\end{abstract}

Cell Death and Differentiation (2009) 16, 984-990; doi:10.1038/cdd.2009.54; published online 8 May 2009

When eukaryotic cells face challenging conditions such as nutrient deficiency, they degrade a portion of their own constituents by inducing a cellular process called autophagy. ${ }^{1}$ Among the several modes of autophagy, we will focus on macroautophagy in this review; this process involves a unique membrane structure termed the autophagosome. Autophagosomes are spherical structures consisting of double bilayered membranes with diameters of about $500 \mathrm{~nm}$. In the cultured mammalian cells, tens or hundreds of autophagosomes are formed in the cytoplasm within a few hours of starvation; these autophagosomes are subsequently delivered to the perinuclear region where they fuse with lysosomes. The contents of the autophagosome are then degraded by lysosomal hydrolases. The degradation products are primarily needed to supply nutrients, and it has recently become evident that autophagy is involved in a number of physiological phenomena. $^{2}$

The mechanism of autophagosome biogenesis is an interesting cell biological problem because it stands apart from the canonical vesicular trafficking process. ${ }^{3}$ In autophagy, a membrane sac called the isolation membrane/ phagophore extends around the degradation substrates, and ultimately fuses to become the autophagosome (Figure 1a). A key breakthrough in this enigma was brought about by the power of yeast genetics, which allowed the identification of more than 10 genes responsible for autophagosome formation through complementation screening. ${ }^{4}$ This collection of genes is now termed Atg, and their orthologues are essentially conserved throughout eukaryotes. Extensive analysis of the Atg genes has been performed and the molecular mechanisms of autophagosome formation are gradually being elucidated. However, relatively less attention has been paid to the late stages of autophagy in comparison to the earlier stages. Here, we will review recent advances in our understanding of the autophagic process, particularly focusing on the later stages, the process between closure of the autophagosome to fusion with the lysosome. It should be noted that closing step has not been well described so far possibly because there has not been a mutant specifically defective in this step. For the rest of autophagic process such as induction mechanism, initiation of the formation, disintegration of autophagic body and recycling of degradation products, please refer the other reviews. $3,5,6$

\section{LC3: An Important Player In Autophagosome Closure?}

Atg8 is an ubiquitin-like protein in yeast and its mammalian orthologues are LC3, GATE-16 and GABARAP. ${ }^{7}$ The roles of LC3 and its paralogues (termed LC3s here) in mammalian autophagy have been obscure primarily because the presence of multiple paralogues has precluded knockdown/out. Recently, a novel method to functionally eliminate the LC3 protein together with its paralogues has been established. ${ }^{8}$ This method employs the exogenous overexpression of an inactive form of Atg4, a cysteine protease that processes Atg8/LC3s. Atg4 cleaves two different targets from Atg8/ LC3s: an unnecessary C-terminal tail sequence and PE, which is conjugated to LC3 by a ubiquitination-like reaction (Figure 1b). ${ }^{9,10}$ Atg4 action on LC3s exposes a glycine residue at the $\mathrm{C}$-terminus and results in form $\mathrm{I}$, unconjugated soluble form that diffuses throughout the cytoplasm. During autophagy, form-I Atg8/LC3 becomes PE-conjugated (form-II) and membrane bound. This process is catalyzed by a ubiquitination-like reaction performed by an E1-like enzyme

\footnotetext{
${ }^{1}$ Department of Cellular Regulation, Research Institute for Microbial Diseases, Osaka University, 3-1 Yamadaoka, Suita, Osaka 565-0871, Japan

${ }^{*}$ Corresponding author: T Yoshimori, Department of Cellular Regulation, Research Institute for Microbial Diseases, Osaka University, 3-1 Yamadaoka, Suita, Osaka, 565-0871, Japan. Tel: + 8166879 8294; Fax: + 8166879 8295; E-mail: tamyoshi@ biken.osaka-u.ac.jp

Keywords: autophagy; autophagosome; membrane fusion; LC3; Atg16 L

Abbreviations: PE, phosphatidylethanolamine; NSF, NEM sensitive factor; SNARE, soluble NSF attachment protein receptors; HA, hemagglutinin; GFP, green fluorescent protein

Received 11.2.09; revised 31.3.09; accepted 20.4.09; Edited by M Piacentini; published online 08.5.09
} 


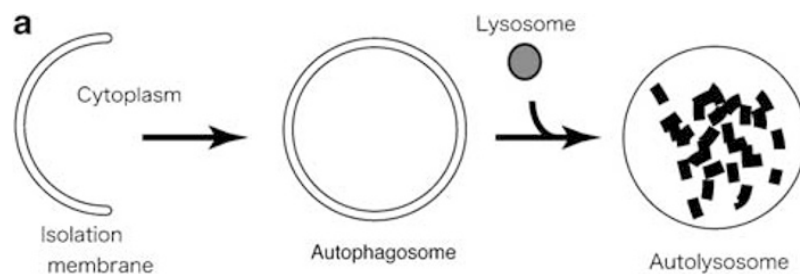

b Pro-LC3

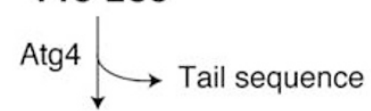

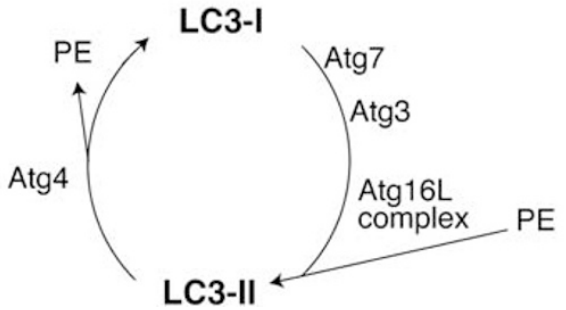

Figure 1 Schematic diagram of autophagy and the LC3 reaction pathway. (a) Representative stages of autophagy. The isolation membrane/phagophore traps a portion of cytosol and becomes an autophagosome. The autophagosome fuses with the lysosome to become an autolysosome and its contents are degraded. (b) The LC3 reaction pathway. LC3 translated from mRNA is called pro-LC3 and possesses an unnecessary C-terminal tail. Subsequently, Atg4 protease cleaves the tail sequence to generate form-I LC3, which has a C-terminal glycine. Form-I LC3 is activated by Atg7 (E1) to become a high-energy thio-ester intermediate conjugated to Atg3 (E2) enzyme. Atg3-LC3 conjugate is recruited to the site where lipidation occurs by directly binding to Atg16 $\mathrm{L}$ complex, and the C-terminus of LC3 forms an amino bond to the head group of phosphatidylethanolamine (PE), generating formII. Form-II LC3 becomes membrane bound. Form-II LC3 is then cleaved at the PE by Atg 4 to regenerate form-I

(Atg7) and an E2-like enzyme (Atg3) (Figure 1b). ${ }^{11}$ The exogenously overexpressed Atg4 inactive mutant has an interesting property: it strongly binds to its product, unconjugated LC3s (form I). The Atg4-bound LC3s cannot be converted to the PE-conjugated form because access to the E1 enzyme is prevented. Therefore, if inactive Atg4 is present in excess of all LC3s in a cell, this will result in the loss of LC3 paralogue function.

As expected, autophagic activity was completely abolished in cells with inactive Atg4 overexpression. ${ }^{8}$ Unexpectedly, however, autophagosome formation was not completely abolished. Electron microscopy showed that many autophagosome-like structures accumulated in the cytosol compared with wild-type cells. Further, about half of these structures were mostly, but not completely, closed. This percentage is likely an underestimation of actual unclosed autophagosome number, as the electron microscopic sections would not necessarily traverse the unclosed regions. These results imply that a defect in LC3s function leads to the failure of autophagosome closure. Essentially similar phenotypes were recapitulated in an Atg3 knockout mouse, in which the conjugating enzyme (E2) involved in the ubiquitination-like lipidation reaction of LC3s is eliminated. ${ }^{12}$ Abnormally elongated isolation membranes were sometimes observed in Atg3 KO cells, and this may be the consequence of failure to terminate elongation because of the absence of LC3s function. ${ }^{12}$ Thus, form-II LC3s seem to be necessary for autophagosome closure.

What kind of membrane dynamics underlie the closing autophagosome? Closure does not occur through typical membrane fusion, as the general membrane fusion facilitator, NSF/Sec18, has been shown to be dispensable for autophagosome formation in yeast. ${ }^{13}$ However, there are interesting similarities between autophagosome closure and the membrane dynamics of cellular infection by the influenza virus. After attaching to the surface of the host cell, influenza virus enters through the endocytic pathway (Figure 2). Once at the endosome, the viral membrane merges with the endosome membrane and the nucleocapsid escapes into the cytosol. This process is catalyzed by the viral HA protein. ${ }^{14}$ When exposed to the acidic environment of the endosome, $\mathrm{HA}$, whose each terminus is inserted into each membrane, undergoes a conformational change that bends two $\alpha$-helical stretches and generates a driving force for membrane fusion. These dynamics represent typical membrane fusion, and in fact, HA structurally resembles the SNARE proteins that are involved in membrane fusion during vesicular trafficking. In the prevailing model of lipid bilayer vesicle fusion (the stalkpore fusion model), ${ }^{15}$ a fusion pore is ultimately generated (Figure 2). On the other hand, during the process of autophagosome formation, the leading edge of the isolation membrane gradually narrows and finally forms a pore. In this analogy, the state that the pre-fused influenza virus is inside the endosome can be regarded as the completely enclosed autophagosome; the endosome membrane corresponds to the outer autophagosome membrane and the influenza membrane corresponds to the inner autophagosome membrane. The state just after HA-mediated fusion between the viral membrane and the endosome can be thought of as analogous to that of the forming autophagosome just about to close. Thus, in terms of membrane dynamics, autophagosome closure proceeds in reverse order of the membrane fusion process in influenza escape (Reverse fusion model). To further clarify this comparison, we will raise a similarity to the multivesicular body, a type of endosome that includes a number of internal vesicles. These internal vesicles are generated through invagination of limiting membrane of the multivesicular body and the final scission. ${ }^{16}$ The membrane dynamics of scission are topologically similar to autophagosome closure. In maturing dendritic cells, the internal vesicles harboring $\mathrm{MHC}$ class II molecules in multivesicular body are competent in back fusion to limiting membrane to transform into long tubules, which are delivered to the plasma membrane. ${ }^{17}$ This back fusion is also topologically similar to viral escape and the reverse of 'Reverse fusion'.

How are the LC3s involved in autophagosome closure? The yeast Atg8 has some interesting capacities regarding membrane dynamics. In vitro reconstitution of the ubiquitinationlike conjugation of PE to Atg8 to form Atg8-PE has been accomplished. ${ }^{18}$ The resulting liposomes harboring Atg8-PE cluster together in the experimental tube, and this is owing to the nature of the Atg8 protein. ${ }^{19}$ Atg8-PE homo-oligomerizes and can catalyze tethering and hemifusion of membrane vesicles. Hemifusion is a type of fusion that merges only the outer leaflets of both pairs of double membranes. However, how hemifusion is involved in autophagosome formation is not 


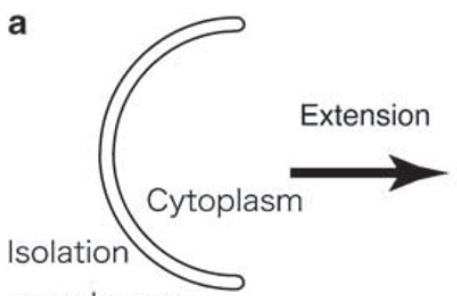

membrane

b
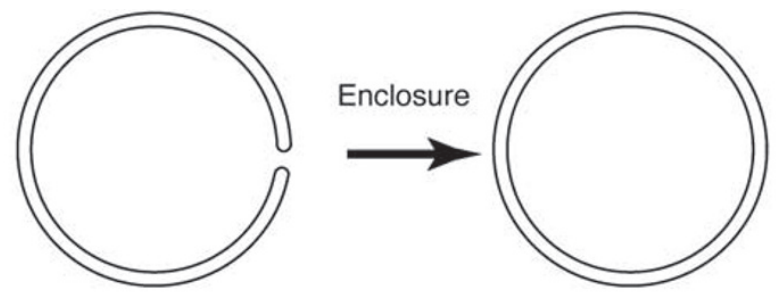

Autophagosome

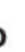

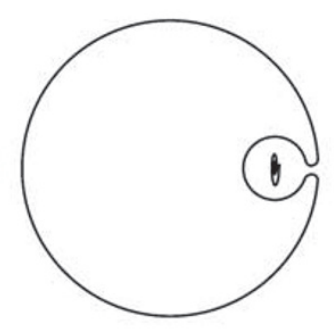

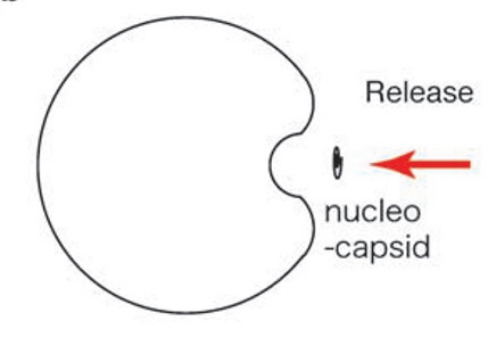

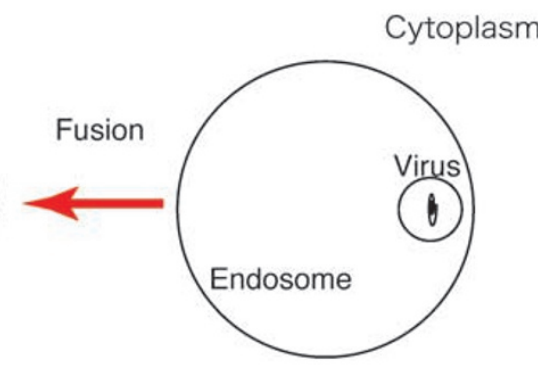

c
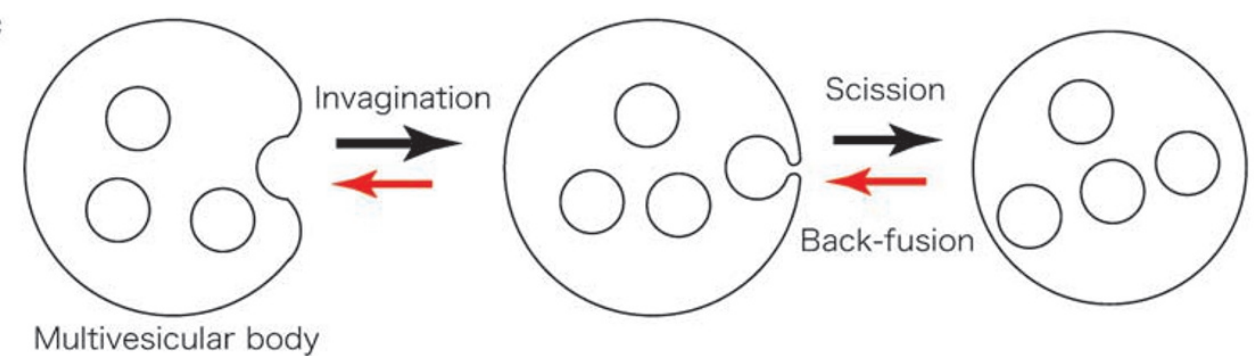

Figure 2 Similarity between autophagosome closure, virus escape and multivesicular body. (a) The isolation membrane/phagophore extends and the leading edge finally closes to complete autophagosome formation. (b) Influenza virus fuses with the endosome and the nucleocapsid is released into the cytoplasm. Topologically, similar membrane structures are found in the events during influenza virus escape from the endosome and in autophagosome formation. However, they occur in reverse order. (c) Multivesicular body generation and back fusion of internal vesicles. Internal vesicles are generated through invagination of the limiting membrane and subsequent scission. In dendritic cells, internal vesicles can back fuse with the limiting membrane

well understood from the results in yeast. The defect seen in LC3s functional deficiency in mammalian cells may show this missing link.

The most well-accepted model of membrane fusion suggests that fusion is initiated by hemifusion, and a stalk connecting the two membranes is then generated (Figure 3a). A fusion pore then opens, leading to the complete fusion. In the reverse model suggested for autophagosome closing, the pore becomes a stalk. In this transformation, a lipid monolayer within a pore disappears (Figure $3 b$ ). This disappearance might be catalyzed by LC3s, because it is similar to the hemifusion process in the sense that opposing lipid molecules in the outer leaflets mix together. The mechanism of progression from stalk to complete closure is not well understood, but it is probable that once the outer membrane is closed and becomes spherical, it is thermodynamically stable enough to generate the force to eliminate the stalk in the inner membrane.

This role for LC3s had not been anticipated from early studies. As the Atg8/LC3s are conjugated to a lipid molecule and then detached from it, one favored model is that these proteins carry lipids to forming autophagosomes. ${ }^{20}$ This model hypothesizes that LC3 is lipidated at some other location and is delivered to the isolation membrane. Although this possibility has not been excluded, recent progress in understanding other Atg proteins fit a new model that LC3s are directly involved in autophagosome closure, at least in mammalian cells. Atg12 is another ubiquitin-like molecule and is conjugated to the central region of the Atg5 protein through its C-terminus. ${ }^{21}$ Atg16 binds to Atg5 at its N-terminal region and forms homo-oligomers through its coiled-coil region. ${ }^{22}$ Therefore, Atg12-Atg5 conjugate and Atg16 (Ag16L in mammalian cells) forms a multimeric super-complex, termed the Atg16 L complex. ${ }^{23}$ The Atg16 L complex has a role as an E3 ligase-like molecule in the LC3s ubiquitination-like reaction, because (i) Atg12 associates with Atg3, an E2-like enzyme involved in the LC3s ubiquitination-like reaction, (ii) Inhibition of membrane recruitment of Atg16L complex leads to a defect in the lipidation reaction and (iii) Atg16L complex artificially localized to the plasma membrane catalyzed the lipidation reaction there. ${ }^{24}$ Thus, the Atg16L complex is competent for the recruitment of the activated E2-LC3s intermediate to its substrate (membrane structures containing PE). Moreover, when yeast Atg12-Atg5 complex is 
a

Viral membrane

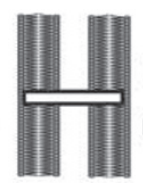

Endosomal

membrane

b Inner

autophago

-somal

membrane

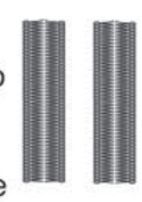

Outer

autophago

-somal

membrane

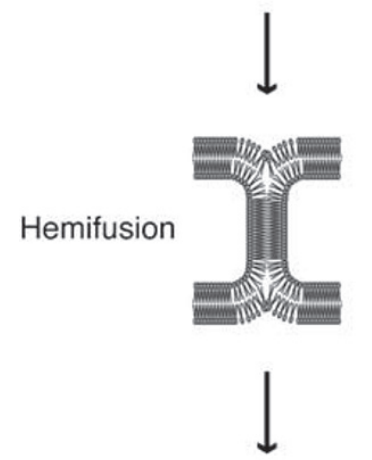

Stalk
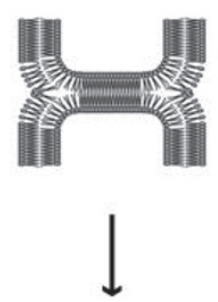

Fusion

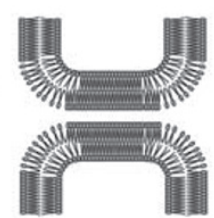

pore

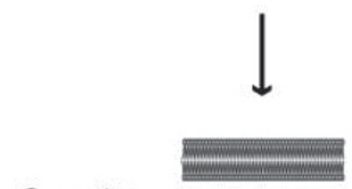

Complete

fusion

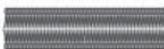

Figure 3 Hypothetical model of LC3-mediated autophagosome closure. (a) The stalk-pore model of HA mediated viral-endosomal membrane fusion. The white rectangle represents HA. HA is inserted into both the viral and endosomal membranes, and by the force generated from HA bending, the membranes undergo hemifusion. Then, a stalk is generated and becomes a membrane pore. Widening of the pore leads to membrane fusion. (b) A hypothetical model of LC3-mediated autophagosome closure. The white rectangle represents $\mathrm{LC} 3$, and the events are depicted from bottom to top. As a result of extension, the leading edge of the isolation membrane converges to a hole narrow enough that LC3 molecules meet. LC3 catalyzes the mixture of the lipids in the cytoplasmic leaflet of the hole, and a stalk forms. The stalk may be disintegrated spontaneously to complete autophagosome formation

added to the Atg8 in vitro reaction system, the reaction is accelerated. ${ }^{25}$ Hence, localization of the Atg16L complex is a critical determinant of where the lipidation reaction occurs. To date, membrane association of Atg16 L complex has been described only at the surface of the isolation membrane, therefore it seems reasonable to speculate that LC3 lipidation occurs at the surface of the isolation membrane (Figure 4). ${ }^{26}$
Atg8 and LC3 are localized on both the inner and outer sides of forming autophagosomes, ${ }^{27,28}$ implying that lipidated Atg8/ LC3s laterally diffuse to traverse to the inner side of isolation membranes. If lipidation takes place on the isolation membrane, it follows that lipidated LC3s function is restricted to the isolation membrane. During lateral diffusion, LC3-PE should traverse the leading edge. Once the isolation membrane 


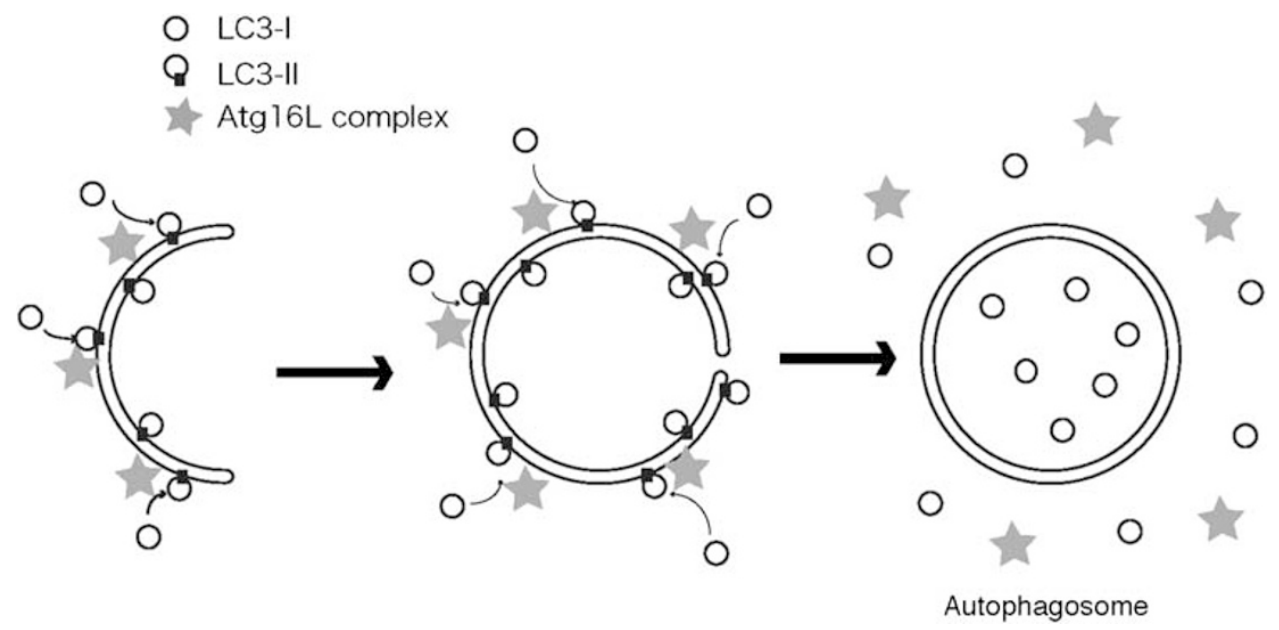

Figure 4 A model of dynamics of Atg16L complex and LC3s. The dynamics of the Atg16L complex and LC3s during autophagosome formation. Star: Atg16 complex. White circle: LC3 form-I. White circle associated with black patch: LC3 form-II. On the basis of a report that Atg16L complex determines the site of the LC3 lipidation reaction, it is postulated that the reaction occurs on the outer surface of forming autophagosomes. LC3s may function at the verge of the membrane in closure. The detachment of LC3/Atg8 from the inner autophagosomal membrane has been clearly shown only in yeast

extends and the leading edge narrows, the LC3-PE will have an opportunity to tether other LC3-PE, and the closure reaction will take place.

It should be noted that the direct involvement of LC3s in autophagosome closure has not been demonstrated and other models might be applicable; for example, Atg16L complex or some other factor may be associated with closure because the behavior of the Atg16L complex is also affected (see below). Moreover, it is still unclear whether this model is also applicable to yeast, and it is highly probable that some differences exist. Even in atg8 null mutant yeast, unclosed autophagosomes like those seen in Atg4 inactive mutantexpressing mammalian cells were not observed by the electron microscopy. ${ }^{27}$ Moreover, Atg8 function is thought to be related to determine of the size of autophagosomes. An Atg8 point mutant that has completely lost hemifusion activity was completely defective in autophagy, but cells with an Atg8 mutant partially defective for hemifusion produces smaller autophagosomes. ${ }^{19}$ Furthermore, artificial reduction of Atg8 expression levels led to the decreased autophagic activity because of the production of smaller autophagosome rather than fewer autophagosomes. ${ }^{29}$ Immuno-EM analysis showed Atg8 signal in the vicinity of the leading edge of forming autophagosomes. ${ }^{27}$ This area may represent the intermediate involved in autophagosome formation. Altogether, yeast Atg8-PE may have additional roles in elongation of the autophagic membrane.

\section{What happens next?}

Atg16L complex is recruited to the forming autophagosome. ${ }^{23}$ Interestingly, GFP-tagged Atg5, representing the Atg16L complex, detaches from the autophagosome and is released into the cytoplasm once the leading edges become continuous and encircled (Figure 4). ${ }^{26}$ Recruitment of GFP-Atg5 to forming autophagosomes occurred normally in cells expressing inactive Atg4, indicating that it does not depend on LC3s function. However, in the presence of inactive Atg4, GFP-Atg5 associates with forming autophagosomes for much longer (greater than $20 \mathrm{~min}$ ) than under normal conditions, in which Atg5 dissociates from forming autophagosomes within $\sim 5$ min of autophagosome completion. ${ }^{8}$ This indicates that detachment of the Atg16 complex is affected by, or possibly dependent on, LC3s function. The direct trigger of this final detachment is still mysterious. The curvature of the autophagosome membrane is one candidate, but this seems less likely, as the size of unclosed autophagosomes is not significantly different from the size of completed autophagosomes in Atg4 inactive mutant-expressing cells. ${ }^{8}$ The closure event itself or LC3s involved in closure may provide a signal that may be transmitted to Atg16L complex spread over the entire autophagosome, even distant from the closure spot. Whether Atg16L complex is localized to the outer surface of forming autophagosomes in yeast, like mammalian Atg16L, remains to be determined. Quantification of Atg16 associated with forming autophagosomes in yeast suggested that there is too little to cover the entire autophagosome as a coat-like clathrin in endocytic vesicles. ${ }^{30}$

Live imaging analysis showed that in the process of single autophagosome formation, the intensity of GFP-Atg8 fluorescent signal at the site of autophagosome formation (termed PAS in yeast) changes in a biphasic manner: it increases gradually and then decreases gradually. ${ }^{30}$ ImmunoEM against Atg8 in yeast showed heavy labeling of the membranes of forming autophagosomes, but less labeling of completed autophagosomes. ${ }^{27}$ Instead, dense Atg8 signal was found evenly in the cytosol incorporated in the autophagic body, the single membrane structure released into the vacuole (lysosome in yeast) after fusion. ${ }^{27}$ In mammalian cells, once the autophagosome fuses with the lysosome, GFP-LC3 signal is no longer detectable, because GFP signal is not emitted owing to the acidic and degradative environment of lysosomes. ${ }^{31}$ GFP-LC3 attached to the outer autophagosome must also disappear. These results indicate that lipidated Atg8/LC3s are liberated from the autophagosome membrane by the action of Atg4 proteases. When does 
this cleavage occur? One possibility is that cleavage occurs constitutively at the surface of the isolation membrane even during the formation period, but the lipidation reaction exceeds the rate of cleavage, so progression of lipidation is apparent. Another possibility is that the cleavage is regulated and occurs only after the completion of autophagosome formation. Absence of this cleavage did not completely abolish autophagic activity, but net autophagic activity was lowered, therefore this cleavage is required in some way in yeast. $^{9}$ One possibility is that if Atg8-PE remains on the surface of the completed autophagosome, it may promote hemifusion between different autophagosomes, and result in failure of vacuolar delivery.

\section{Transport and Fusion}

When observed by live imaging, GFP-Atg5-positive particles, representing forming autophagosomes, move randomly in the vicinity of the initial appearance site. ${ }^{32}$ GFP-LC3-positive particles also show this random initial movement, and then move linearly toward the perinuclear region where lysosomes are localized. ${ }^{32}$ This linear movement utilizes microtubules as tracks and dynein as a motor. ${ }^{32,33}$ GFP-Atg5-positive particles do not show this linear movement; therefore, there must be a regulation to ensure that only autophagosomes engage in this movement. One possibility is that dynein is recruited after the Atg16 $\mathrm{L}$ complex is detached, but the details remain to be elucidated. Involvement of LC3 in this regulation is an interesting possibility, as it was originally identified as microtubule-associated protein light chain $3 .^{34}$ Dyneindependent autophagosome delivery is a physiologically significant process, as it allows the efficient delivery of degradation substrates captured throughout the cytoplasm to the lysosomes. This significance is evident also from the study on LAMP-2, which is a lysosomal membrane protein and its absence is causative of the Danon disease, an $\mathrm{X}$-linked dominant disorder that includes hypertrophic cardiomyopathy, skeletal muscle myopathy and variable mental retardation. ${ }^{35}$ In the LAMP-2 knockout mouse, lysosomes are not concentrated in the perinuclear region but are dispersed throughout the cytoplasm; therefore, autophagosomes cannot fuse to lysosome and accumulate in the cytoplasm. ${ }^{35}$

The lysosome is the final destination of autophagosomes and the outer membrane of autophagosomes ultimately fuses with the lysosomal membrane. The fused organelle is called the autolysosome and this process is also referred to as autophagosome maturation. This fusion process has been analyzed, in detail, in yeast as autophagosome to vacuole fusion. In this system, fusion is dependent on the canonical fusion machinery, the Rab-SNARE system, and the set of involved molecules are thought to be almost identical to those involved in vacuole-vacuole homotypic fusion. ${ }^{36} \mathrm{NSF} / \mathrm{Sec} 18$, which is generally involved in vesicle fusion, is also required for autophagosome vacuole fusion. ${ }^{13}$ Ypt7 is a Rab small GTPase involved both in autophagosome-vacuole and vacuole-vacuole fusion. ${ }^{27}$ The Ccz1-Mon1 protein complex acts together with Ypt7 in these fusion processes. ${ }^{37-39}$ The Ypt7 homologue, Rab7, has been shown to be involved in autophagosome-lysosome fusion in mammalian cells, ${ }^{31,40,41}$ Vam3, Vam7 and Vti1 are SNARE proteins involved in fusion in yeast. ${ }^{42,43}$ The key question remaining to be answered is what is the SNARE molecule residing in autophagosomes (corresponding to $\mathrm{v}$-SNARE) and how it is delivered. The current SNARE model ${ }^{44}$ predicts at least one SNARE molecule is localized in autophagosome. If it is a tail-anchored transmembrane protein, as are the majority of the SNARE family, we would predict the existence of a vesicular trafficmediated connection to an unknown source membrane. In this regard, it should be noted that classical morphological studies showed the existence of a hybrid organelle of autophagosomes and early/late endosomes, termed amphisomes. ${ }^{45}$ The SNARE molecule involved in lysosomal fusion may be delivered through amphisome formation, but this would still leave the question of how the SNARE involved in amphisome fusion is delivered. To date, no specific v-SNARE has been assigned as such a candidate, and identification of the v-SNARE would give us a critical clue to the identity of the source of the autophagosome membrane. It would also be interesting to determine when the SNARE molecule is recruited to the autophagosome, because there should be some mechanism to prevent fusion of lysosomes and preenclosed autophagosomes if the SNARE is already recruited. Class C Vps/HOPS proteins consist of a tethering protein complex including an SM (Sec1/Munc18-like) protein and a Rab GEF (GDP/GTP exchanger) for Ypt7, and are involved in fusion. ${ }^{36,46}$ Recently, it was found that the mammalian HOPS complex binds to UVRAG, which is a Beclin-1 binding protein. ${ }^{47}$ Beclin-1, the mammalian homologue of yeast Atg6, also interacts with class III phosphatidylinositol 3-kinase/ hVps34 and is essential for autophagosome formation. ${ }^{48,49}$ Overexpression of UVRAG promotes autophagosome-lysosome fusion; therefore, it is possible that Beclin-1 is a facilitator of the fusion process in addition to its role in autophagosome formation. ${ }^{47}$

\section{Future Direction}

In this review, we discussed the late stages of autophagy and proposed novel model in autophagosome closure. It is no doubt that a number of molecules link each stage to faithfully conduct the dynamic membrane rearrangement. One of such molecules, but its role in the late stages is totally unknown, is phosphatidylinositol 3-phosphate. It has been established that phosphatidylinositol 3-phosphate plays a pivotal role in at least in the early stages of autophagy. ${ }^{50}$ The yeast autophagosome is enriched for phosphatidylinositol 3-phosphate, but it is not clear if this is the case for mammalian autophagosomes. ${ }^{51}$ Recently, it has been shown that a specific subdomain of the ER (termed the omegasome) becomes deformed to overlap with the nascent autophagosome, and autophagosome formation takes place inside it. The omegasome is enriched for phosphatidylinositol 3-phosphate and interestingly, the omegasome and/or phosphatidylinositol 3-phosphate disappears once the autophagosome is generated. How phosphatidylinositol 3-phosphate is involved in the late stages will be important to the further understanding of the autophagy regulation.

1. Levine B, Klionsky DJ. Development by self-digestion: molecular mechanisms and biological functions of autophagy. Dev Cell 2004; 6: 463-477. 
27. Kirisako T, Baba M, Ishihara N, Miyazawa K, Ohsumi M, Yoshimori T et al. Formation process of autophagosome is traced with Apg8/Aut7p in yeast. J Cell Biol 1999; 147: 435-446.

cellular self-digestion. Nature 2008; 451: 1069-1075.

3. Xie Z, Klionsky DJ. Autophagosome formation: core machinery and adaptations. Nat Cell Biol 2007; 9: 1102-1109.

4. Suzuki K, Ohsumi Y. Molecular machinery of autophagosome formation in yeast, Saccharomyces cerevisiae. FEBS Lett 2007; 581: 2156-2161.

5. Yorimitsu T, Klionsky DJ. Autophagy: molecular machinery for self-eating. Cell Death Differ 2005; 12 (Suppl 2): 1542-1552.

6. Mizushima N. Autophagy: process and function. Genes Dev 2007; 21: 2861-2873.

7. Geng J, Klionsky DJ. The Atg8 and Atg12 ubiquitin-like conjugation systems in macroautophagy. 'Protein modifications: beyond the usual suspects' review series. EMBO Rep 2008; 9: 859-864.

8. Fujita N, Hayashi-Nishino M, Fukumoto H, Omori H, Yamamoto A, Noda T et al. An Atg4B mutant hampers the lipidation of LC3 paralogues and causes defects in autophagosome closure. Mol Biol Cell 2008; 19: 4651-4659.

9. Kirisako T, Ichimura Y, Okada H, Kabeya Y, Mizushima N, Yoshimori T et al. The reversible modification regulates the membrane-binding state of Apg8/Aut7 essential for autophagy and the cytoplasm to vacuole targeting pathway. J Cell Biol 2000; 151: 263-276.

10. Kabeya Y, Mizushima N, Yamamoto A, Oshitani-Okamoto S, Ohsumi Y, Yoshimori T. LC3, GABARAP and GATE16 localize to autophagosomal membrane depending on form-II formation. J Cell Sci 2004; 117 (Part 13): 2805-2812.

11. Ichimura $Y$, Kirisako $T$, Takao $T$, Satomi $Y$, Shimonishi $Y$, Ishihara $N$ et al. A ubiquitin-like system mediates protein lipidation. Nature 2000; 408: 488-492.

12. Sou YS, Waguri S, Iwata J, Ueno T, Fujimura T, Hara T et al. The Atg 8 conjugation system is indispensable for proper development of autophagic isolation membranes in mice. Mol Biol Cell 2008; 19: 4762-4775.

13. Ishihara N, Hamasaki M, Yokota S, Suzuki K, Kamada Y, Kihara A et al. Autophagosome requires specific early Sec proteins for its formation and NSF/SNARE for vacuolar fusion. Mol Biol Cell 2001; 12: 3690-3702.

14. Bentz J, Mittal A. Architecture of the influenza hemagglutinin membrane fusion site. Biochim Biophys Acta 2003; 1614: 24-35.

15. Chernomordik L, Kozlov MM, Zimmerberg J. Lipids in biological membrane fusion. J Membr Biol 1995; 146: 1-14.

16. Katzmann DJ, Odorizzi G, Emr SD. Receptor downregulation and multivesicular-body sorting. Nat Rev Mol Cell Biol 2002; 3: 893-905.

17. Murk JL, Stoorvogel W, Kleijmeer MJ, Geuze HJ. The plasticity of multivesicular bodies and the regulation of antigen presentation. Semin Cell Dev Biol 2002; 13: 303-311.

18. Ichimura $Y$, Imamura $Y$, Emoto $K$, Umeda $M$, Noda $T$, Ohsumi $Y$. In vivo and in vitro reconstitution of Atg8 conjugation essential for autophagy. J Biol Chem 2004; 279: 40584-40592.

19. Nakatogawa $\mathrm{H}$, Ichimura $\mathrm{Y}$, Ohsumi $\mathrm{Y}$. Atg8, a ubiquitin-like protein required for autophagosome formation, mediates membrane tethering and hemifusion. Cell 2007; 130: 165-178

20. Noda T, Suzuki K, Ohsumi Y. Yeast autophagosomes: de novo formation of a membrane structure. Trends Cell Biol 2002; 12: 231-235.

21. Mizushima N, Noda T, Yoshimori T, Tanaka Y, Ishii T, George MD et al. A protein conjugation system essential for autophagy. Nature 1998; 395: 395-398.

22. Mizushima N, Noda T, Ohsumi Y. Apg16p is required for the function of the Apg12p-Apg5p conjugate in the yeast autophagy pathway. EMBO J 1999; 18: 3888-3896.

23. Mizushima N, Kuma A, Kobayashi Y, Yamamoto A, Matsubae M, Takao T et al. Mouse Apg16L, a novel WD-repeat protein, targets to the autophagic isolation membrane with the Apg12-Apg5 conjugate. J Cell Sci 2003; 116 (Part 9): 1679-1688.

24. Fujita N, Itoh T, Omori H, Fukuda M, Noda T, Yoshimori T. The Atg16 complex specifies the site of LC3 lipidation for membrane biogenesis in autophagy. Mol Biol Cell 2008; 19 2092-2100.

25. Hanada T, Noda NN, Satomi Y, Ichimura Y, Fujioka Y, Takao T et al. The Atg12-Atg5 conjugate has a novel E3-like activity for protein lipidation in autophagy. J Biol Chem 2007; 282: 37298-37302.

26. Mizushima N, Yamamoto A, Hatano M, Kobayashi Y, Kabeya Y, Suzuki K et al. Dissection of autophagosome formation using Apg5-deficient mouse embryonic stem cells. J Cell Biol 2001; 152: 657-668
28. Kabeya $Y$, Mizushima N, Ueno T, Yamamoto A, Kirisako T, Noda T et al. LC3, a mammalian homologue of yeast Apg8p, is localized in autophagosome membranes after processing. EMBO J 2000; 19: 5720-5728.

29. Xie Z, Nair U, Klionsky DJ. Atg8 controls phagophore expansion during autophagosome formation. Mol Biol Cell 2008; 19: 3290-3298.

30. Geng J, Baba M, Nair U, Klionsky D. Quantitative analysis of autophagy-related protein stoichiometry by fluorescence microscopy. J Cell Biol 2008; 182: 129-140.

31. Kimura S, Noda T, Yoshimori T. Dissection of the autophagosome maturation process by a novel reporter protein, tandem fluorescent-tagged LC3. Autophagy 2007; 3: 452-460.

32. Kimura S, Noda T, Yoshimori T. Dynein-dependent movement of autophagosomes mediates efficient encounters with lysosomes. Cell Struct Funct 2008; 33: 109-122.

33. Fass E, Shvets E, Degani I, Hirschberg K, Elazar Z. Microtubules support production of starvation-induced autophagosomes but not their targeting and fusion with lysosomes. J Biol Chem 2006; 281: 36303-36316.

34. Kuznetsov SA, Gelfand VI. $18 \mathrm{kDa}$ microtubule-associated protein: identification as a new light chain (LC-3) of microtubule-associated protein 1 (MAP-1). FEBS Lett 1987; 212 145-148.

35. Saftig P, Beertsen W, Eskelinen EL. LAMP-2: a control step for phagosome and autophagosome maturation. Autophagy 2008; 4: 510-512.

36. Ostrowicz CW, Meiringer CT, Ungermann C. Yeast vacuole fusion: a model system for eukaryotic endomembrane dynamics. Autophagy 2008; 4: 5-19.

37. Kucharczyk R, Kierzek AM, Slonimski PP, Rytka J. The Ccz1 protein interacts with Ypt7 GTPase during fusion of multiple transport intermediates with the vacuole in S.cerevisiae. J Cell Sci 2001; 114 (Part 17): 3137-3145

38. Meiling-Wesse K, Barth $\mathrm{H}$, Thumm M. Ccz1p/Aut11p/Cvt16p is essential for autophagy and the cvt pathway. FEBS Lett 2002; 526: 71-76.

39. Wang CW, Stromhaug PE, Shima J, Klionsky DJ. The Ccz1-Mon1 protein complex is required for the late step of multiple vacuole delivery pathways. J Biol Chem 2002; 277 47917-47927.

40. Gutierrez MG, Munafo DB, Beron W, Colombo Ml. Rab7 is required for the norma progression of the autophagic pathway in mammalian cells. J Cell Sci 2004; 117 (Part 13): 2687-2697.

41. Jager S, Bucci C, Tanida I, Ueno T, Kominami E, Saftig P et al. Role for Rab7 in maturation of late autophagic vacuoles. J Cell Sci 2004; 117 (Part 20): 4837-4848.

42. Darsow T, Rieder SE, Emr SD. A multispecificity syntaxin homologue, Vam3p, essential for autophagic and biosynthetic protein transport to the vacuole. J Cell Biol 1997; 138: 517-529.

43. Fischer von Mollard G, Stevens TH. The Saccharomyces cerevisiae v-SNARE Vti1p is required for multiple membrane transport pathways to the vacuole. Mol Biol Cell 1999; 10 : $1719-1732$.

44. Sudhof TC, Rothman JE. Membrane fusion: grappling with SNARE and SM proteins. Science 2009; 323: 474-477.

45. Fader CM, Colombo MI. Autophagy and multivesicular bodies: two closely related partners. Cell Death Differ 2009; 16: 70-78.

46. Rieder SE, Emr SD. A novel RING finger protein complex essential for a late step in protein transport to the yeast vacuole. Mol Biol Cell 1997; 8: 2307-2327.

47. Liang C, Lee JS, Inn KS, Gack MU, Li Q, Roberts EA et al. Beclin1-binding UVRAG targets trafficking. Nat Cell Biol 2008; 10: 776-787.

48. Kihara A, Kabeya Y, Ohsumi Y, Yoshimori T. Beclin-phosphatidylinositol 3-kinase complex functions at the trans-Golgi network. EMBO Rep 2001; 2: 330-335.

49. Yue Z, Jin S, Yang C, Levine AJ, Heintz N. Beclin 1, an autophagy gene essential for early embryonic development, is a haploinsufficient tumor suppressor. Proc Natl Acad Sci U S A 2003; 100: 15077-15082

50. Yoshimori T, Noda T. Toward unraveling membrane biogenesis in mammalian autophagy. Curr Opin Cell Biol 2008; 20: 401-407.

51. Obara K, Noda T, Niimi K, Ohsumi Y. Transport of phosphatidylinositol 3-phosphate into the vacuole via autophagic membranes in Saccharomyces cerevisiae. Genes Cells 2008; 13: $537-547$ the class $C$ Vps complex to coordinate autophagosome maturation and endocytic 